Rev. Dr M. Wilcox, The Manse, 47 Richardson Street, Middle Park, S.C. 6, Victoria, Australia.

Prof. A: N. Wrlder, Harvard Divinity School, Andover Hall, Francis Avenue, Cambridge 38, Mass., U.S.A.

Rev. C. S. C. Williams, Merton College, Oxford.

Right Rev. R. R. Williams, Bishop of Leicester, Bishop's Lodge, ro Springfield Road, Leicester.

Rev. Dr R. McL. Wilson, St Mary's College, St Andrews, Fife, Scotland.

* Dr H. G. Wood, 26 Linden Road, Bournville, Birmingham.

Dr Olive Wyon, Cantelowes Cottage, Hinxton, Saffron Walden, Essex.

Rev. J. E. Yates, Bryn Awel, Esplanade, Penmaenmawr, N. Wales.

Prof. Dr J. DE ZwaAn, Boerhaavelaan 3 I, Leiden, Holland.

\title{
OBITUARY
}

We regret to record the death of the Rev. Professor J. Newton Davies, Bournemouth.

\section{OFFICERS AND COMMITTEE MEMBERS} $1957-8$

President: Dr H. G. Wood, 26 Linden Road, Birmingham 30, England.

Secretary: Rev. K. Grayston, 17 Northover Road, Westbury-on-Trym, Bristol, England.

Treasurer: Rev. J. N. Sanders, Peterhouse, Cambridge, England.

\section{Committee Members}

Père P. Benoit, O.P., Ecole Biblique et Archéologique Française, Jerusalem P.O.B. 53 (Arab Zone), Jordan via Amman.

Professor Dr K. G. Kuhn, Friesenberg Ia, Heidelberg, Germany. Professor Dr J. Munck, Studenterkollegierne, Aarhus, Denmark. Professor M. M. Parvis, Emory University, Georgia, U.S.A. Very Rev. Dr E. G. Selwyn, The Deanery, Winchester, England. Professor H. J. Cadbury, 774 Millbrook Lane, Haverford, Pa., U.S.A. Rev. Professor M. Black, St Mary's College, St Andrews, Scotland. Professor Dr E. Schweizer, Pilgerweg 8, Zürich 44, Switzerland. 